\title{
An Alternating Direction Implicit Method for Solving Projected Generalized Continuous-Time Sylvester Equations
}

\author{
Yiqin $\operatorname{Lin}^{a}$, Liang Bao \\ ${ }^{a}$ Department of Mathematics and Computational Science Hunan University of of Science and Engineering \\ Yongzhou 425100, P. R. China \\ ${ }^{b}$ Department of Mathematics East China University of Science and Technology Shanghai, 200237, P. R. China
}

\begin{abstract}
In this paper we propose a low-rank alternating direction implicit (ADI) method to solve projected generalized continuous-time Sylvester equations with low-rank right-hand sides. Such equations arise in control theory including the computation of inner products and $\mathrm{H}_{2}$ norms, and the model reduction based on balanced truncation for descriptor systems. The requirements of this method are moderate with respect to both computational cost and memory. Numerical experiments presented in this paper show the effectiveness of the proposed method.
\end{abstract}

Index Terms: Projected generalized Sylvester equations; Alternating direction implicit method; Low-rank approximate solution; C-stable

(C) 2011 Published by MECS Publisher. Selection and/or peer review under responsibility of the Research Association of Modern Education and Computer Science.

\section{Introduction}

In this paper we consider the projected generalized continuous-time Sylvester equation of the form

$$
\left\{\begin{array}{l}
A X C+D X B+P_{l, 1} E P_{r, 2}=0, \\
X=P_{r, 1} X P_{l, 2},
\end{array}\right.
$$

where $A, D \in \square^{n \times n}, B, C \in \square^{m \times m}, E \in \square^{n \times m}$, and $X \in \square^{n \times m}$ is the sought-after solution. Here, $P_{r, 1}$ and $P_{r, 2}$ are the spectral projectors onto the right deflating subspaces corresponding to the finite eigenvalues of the

* Corresponding author.

E-mail address: yqlin@yahoo.cn, nlbao@yahoo.cn 
pencils $\lambda D-A$ and $\lambda C-B$, respectively, $Q_{r, 1}=I-P_{r, 1}$ and $Q_{r, 2}=I-P_{r, 2}$ are the spectral projectors onto the right deflating subspaces corresponding to the eigenvalue at infinity. The spectral projectors onto the left deflating subspaces corresponding to the finite eigenvalues of $\lambda D-A$ and $\lambda C-B$ are denoted by $P_{l, 1}$ and $P_{l, 2}$, respectively, while $Q_{l, 1}=I-P_{l, 1}$ and $Q_{l, 2}=I-P_{l, 2}$ are the spectral projectors onto the left deflating subspaces corresponding to the eigenvalue at infinity. We assume that the matrices $C$ and $D$ are singular, but the pencils $\lambda D-A$ and $\lambda C-B$ are regular, i.e., $\operatorname{det}(\lambda D-A)$ and $\operatorname{det}(\lambda C-B)$ are not identically zero. Under the assumption, the pencils $\lambda D-A$ and $\lambda C-B$ have the Weierstrass canonical forms [1]: there exist nonsingular $n \times n$ matrices $W_{1}, T_{1}$ and $m \times m$ matrices $W_{2}, T_{2}$ such that

$$
\begin{aligned}
& D=W_{1}\left[\begin{array}{cc}
I & 0 \\
0 & N^{(A)}
\end{array}\right] T_{1}, \quad A=W_{1}\left[\begin{array}{cc}
J^{(A)} & 0 \\
0 & I
\end{array}\right] T_{1}, \\
& C=W_{2}\left[\begin{array}{cc}
I & 0 \\
0 & N^{(B)}
\end{array}\right] T_{2}, \quad B=W_{2}\left[\begin{array}{cc}
J^{(B)} & 0 \\
0 & I
\end{array}\right] T_{2},
\end{aligned}
$$

where $J^{(A)}, J^{(B)}, N^{(A)}$ and $N^{(B)}$ are block diagonal matrices with each diagonal block being a Jordan block. The eigenvalues of $J^{(A)}$ and $J^{(B)}$ are the finite eigenvalues of the pencils $\lambda D-A$ and $\lambda C-B$, respectively. $N^{(A)}$ and $N^{(B)}$ correspond to the eigenvalue at infinity. The index $\gamma$ of nilpotency of $N^{(A)}$ is called the index of the pencil $\lambda D-A$. Using (2) and (3), $P_{l, 1}, P_{l, 2}, P_{r, 1}$ and $P_{r, 2}$ can be expressed as

$$
\begin{aligned}
& P_{l, 1}=W_{1}\left[\begin{array}{ll}
I & 0 \\
0 & 0
\end{array}\right] W_{1}^{-1}, \quad P_{l, 2}=W_{2}\left[\begin{array}{ll}
I & 0 \\
0 & 0
\end{array}\right] W_{2}^{-1}, \\
& P_{r, 1}=T_{1}^{-1}\left[\begin{array}{ll}
I & 0 \\
0 & 0
\end{array}\right] T_{1}, \quad P_{r, 2}=T_{2}^{-1}\left[\begin{array}{ll}
I & 0 \\
0 & 0
\end{array}\right] T_{2} .
\end{aligned}
$$

As $m=n, B=A^{T}$, and $C=D^{T}$, (1) is referred to as the projected generalized continuous-time Lyapunov equation, which arises in stability analysis and control design problems for descriptor systems including the characterization of controllability and observability properties, balanced truncation model order reduction, determining the minimal and balanced realizations as well as computing $\mathrm{H}_{2}$ and Hankel norms; see $[2,3,4,5]$ and the references therein. If the pencil $\lambda D-A$ is c-stable, i.e., all its finite eigenvalues have negative real part, then the projected generalized Lyapunov equation has a unique solution for each $E$, and if, additionally, $E$ is symmetric and positive semidefinite, then the solution $X$ is symmetric and positive semidefinite, see, e.g., [6] for details. Recently, several numerical methods have been proposed in the literature for solving the projected generalized Lyapunov equation. In [7], two direct methods, the generalized BartelsStewart method and the generalized Hammarling method, were proposed for the projected generalized Lyapunov equation. The generalized Hammarling method is designed to obtain the Cholesky factor of the solution. These 
two methods are based on the generalized real Schur form of the pencil $\lambda D-A$, and require $O\left(n^{3}\right)$ flops and $O\left(n^{2}\right)$ memory. Iterative methods to solve the projected generalized Lyapunov equation have also been proposed. Stykel [8] extended the ADI method to the projected equation. Another iterative method for the projected generalized Lyapunov equation is the modified generalized matrix sign function method [9]. Unlike the classical generalized matrix sign function method, the variant converges quadratically independent of the index of the underlying matrix pencil, see [9] for more details.

The projected generalized continuous-time Sylvester equation (1) arises in some applications including the computation of inner products and $H_{2}$ norms, and the balanced truncation model reduction based on Sylvester equation for descriptor systems. It has been shown in [10] that the pencils $\lambda D-A$ and $\lambda C-B$ are c-stable, (1) has a unique solution.

In this paper, we propose a low-rank ADI method for solving the projected generalized Sylvester equation (1). This work presented here is an extension of [8]. Numerical experiments show the effectiveness of the proposed method.

Throughout this paper, we adopt the following notations. The square identity and zero matrices are denoted by $I$ and 0 , respectively. The spaces of $m \times n$ real matrices are denoted by $\square^{m \times n}$. The 2 -norm and the Frobenius matrix norm are denoted by $\|\cdot\|_{2}$ and $\|\cdot\|_{F}$, respectively. The superscript $"{ }^{T} "$ denotes the transposition of a vector or a matrix. The open left and right half-plane are denoted by $C^{-}$and $C^{+}$, respectively. We shall also adopt MATLAB-like convention to access the entries of vectors and matrices. For a matrix $X, X_{(i, j)}$ is $X$,s $(i, j)$ th entry; $X$,s submatrices $X_{(k: l, i: j)}, X_{(k: l,:)}$ and $X_{(:, i: j)}$ consist of intersections of row $k$ to row $l$ and column $i$ to column $j$, row $k$ to row $l$, and column $i$ to column $j$, respectively.

The remainder of the paper is organized as follows. In Section 2, we propose a low-rank alternating direction implicit method for the solution of equation (1). Section 3 is devoted to some numerical tests. Conclusion is given in the last section.

\section{Alternating direction implicit method}

We always assume that the pencils $\lambda D-A$ and $\lambda C-B$ are c-stable, i.e., all their finite eigenvalues have negative real part. Hence, the matrices $A$ and $B$ are nonsingular. Multiplying the first equation in (1) on the left by $A^{-1}$ and on the right by $B^{-1}$, we get the following projected standard Sylvester equation

$$
\left\{\begin{array}{l}
\left(A^{-1} D\right) X+X\left(C B^{-1}\right)+A^{-1} P_{l, 1} E P_{r, 2} B^{-1}=0, \\
X=P_{r, 1} X P_{l, 2} .
\end{array}\right.
$$

The iterates $X_{k}$ of the ADI iteration for (7) are usually generated by the alternating solution of two linear systems with multiple right-hand sides 


$$
\begin{gathered}
\left(A^{-1} D-\beta_{k} I\right) X_{k-\frac{1}{2}}=X_{k-1}\left(C B^{-1}+\beta_{k} I\right)+A^{-1} P_{l, 1} E P_{r, 2} B^{-1}, \\
X_{k}\left(C B^{-1}+\alpha_{k} I\right)=\left(A^{-1} D-\alpha_{k} I\right) X_{k-\frac{1}{2}}-A^{-1} P_{l, 1} E P_{r, 2} B^{-1},
\end{gathered}
$$

where $X_{0}=0$ and the shift parameters

$\left\{\alpha_{i}\right\}_{i=1}^{k}$ and $\left\{\beta_{i}\right\}_{i=1}^{k}$ are elements of $C^{-}$and $C^{+}$, respectively. These two equations are equivalent to the following single iteration step:

$$
\begin{aligned}
X_{k}= & \left(A^{-1} D-\beta_{k} I\right)^{-1}\left(A^{-1} D-\alpha_{k} I\right) X_{k-1} \\
& \times\left(C B^{-1}+\beta_{k} I\right)\left(C B^{-1}+\alpha_{k} I\right)^{-1} \\
& +\left(\beta_{k}-\alpha_{k}\right)\left(A^{-1} D-\beta_{k} I\right)^{-1} \\
& \times A^{-1} P_{l, 1} E P_{r, 2} B^{-1}\left(C B^{-1}+\alpha_{k} I\right)^{-1} .
\end{aligned}
$$

About the ADI iteration, we have the following result.

Theorem II.1. For all $k \geq 0$, the matrix $X_{k}$ defined by the ADI iteration satisfies the second equation in (7), i.e.,

$$
X_{k}=P_{r, 1} X_{k} P_{l, 2}
$$

Proof. The result follows directly from

$$
\begin{aligned}
& P_{r, 1}\left(A^{-1} D-\beta_{k} I\right)^{-1}=\left(A^{-1} D-\beta_{k} I\right)^{-1} P_{r, 1}, \\
& P_{r, 1}\left(A^{-1} D-\alpha_{k} I\right)=\left(A^{-1} D-\alpha_{k} I\right) P_{r, 1}, \\
& \left(C B^{-1}+\alpha_{k} I\right)^{-1} P_{l, 2}=P_{l, 2}\left(C B^{-1}+\alpha_{k} I\right)^{-1}, \\
& \left(C B^{-1}+\beta_{k} I\right) P_{l, 2}=P_{l, 2}\left(C B^{-1}+\beta_{k} I\right) .
\end{aligned}
$$

We can rewrite the iteration (8) as

$$
\begin{aligned}
X_{k}= & \left(D-\beta_{k} A\right)^{-1}\left(D-\alpha_{k} A\right) X_{k-1} \\
& \times\left(C+\beta_{k} B\right)\left(C+\alpha_{k} B\right)^{-1} \\
& +\left(\beta_{k}-\alpha_{k}\right)\left(D-\beta_{k} A\right)^{-1} P_{l, 1} E P_{r, 2}\left(C+\alpha_{k} B\right)^{-1} .
\end{aligned}
$$

Note that $X_{k}$ is explicitly computed by the ADI iteration (9), so the storage requirement is $O(m n)$. One should notice that in many cases the storage requirement is the limiting factor rather than the amount of computation. We note that low-rank schemes are the only existing methods that can effectively solve large-scale Lyapunov/Sylvester equations. Assume that the low-rank right-hand side $E$ has the factored form $E=F G$ with $F \in \square^{n \times r}$ and $G \in \square^{r \times m}$. Instead of explicitly forming the solution $X$, the low-rank method compute and store approximate solutions in low-rank factored form. If the numerical rank $l$ of $X$ is much smaller than $\min \{m, n\}$, i.e. $l \square \min \{m, n\}$, then the storage is reduced from $O(m n)$ to $O(m l)$ or $O(n l)$.

The key idea in the low-rank version of the ADI iteration is to rewrite the iteration $X_{k}$ in (9) as an outer product:

$$
X_{k}=Y_{k} H_{k} Z_{k} \text {. }
$$


This is always possible since starting with the initial guess $X_{0}=0_{n \times m}$. The low-rank alternating direction implicit (LR-ADI) method is based on (9). Replacing $X_{k-1}$ with $Y_{k-1} H_{k-1} Z_{k-1}$, (9) can be reformulated in terms of the low-rank factors as

$$
X_{k}=Y_{k} H_{k} Z_{k}
$$

where

$$
\begin{aligned}
Y_{k} & =\left[\begin{array}{ll}
\left(D-\beta_{k} A\right)^{-1} P_{l, 1} F & \left(D-\beta_{k} A\right)^{-1}\left(D-\alpha_{k} A\right) Y_{k-1}
\end{array}\right], \\
H_{k} & =\left[\begin{array}{cc}
\left(\beta_{k}-\alpha_{k}\right) I & \\
H_{k-1}
\end{array}\right], \\
Z_{k} & =\left[\begin{array}{c}
G P_{r, 2}\left(C+\alpha_{k} B\right)^{-1} \\
Z_{k-1}\left(C+\beta_{k} B\right)\left(C+\alpha_{k} B\right)^{-1}
\end{array}\right] .
\end{aligned}
$$

From the fact that $Y_{0}, H_{0}$ and $Z_{0}$ are all zero

matrices, it can be seen that $Y_{k}$ is $n \times k r, H_{k}$ is $k r \times k r$ and $Z_{k}$ is $k r \times m$. Thus the rank of $X_{k}$ is no more than $k r$. Since the order of the ADI parameters $\left\{\alpha_{i}\right\}_{i=1}^{k}$ and $\left\{\beta_{i}\right\}_{i=1}^{k}$ is not important, the ordering of $\left\{\alpha_{i}\right\}_{i=1}^{k}$ and $\left\{\beta_{i}\right\}_{i=1}^{k}$ can be reversed. As shown in [8], we have the following iterative scheme

where

$$
\begin{aligned}
& Y_{k}=\left[\begin{array}{llll}
Y^{(1)} & Y^{(2)} & \cdots & Y^{(k)}
\end{array}\right], \\
& \left\{\begin{aligned}
Y^{(1)} & =\left(D-\beta_{1} A\right)^{-1} P_{l, 1} F, \\
Y^{(i+1)} & =\left(D-\beta_{i+1} A\right)^{-1}\left(D-\alpha_{i} A\right) Y^{(i)}, \quad i=1,2, \cdots, k-1,
\end{aligned}\right.
\end{aligned}
$$

and

where

$$
Z_{k}=\left[\begin{array}{llll}
Z^{(1)} & Z^{(2)} & \cdots & Z^{(k)}
\end{array}\right]
$$

$$
\left\{\begin{aligned}
Z^{(1)} & =G P_{r, 2}\left(C+\alpha_{1} B\right)^{-1}, \\
Z^{(i+1)} & =Z^{(i)}\left(C+\beta_{i} B\right)\left(C+\alpha_{i+1} B\right)^{-1}, \quad i=1,2, \cdots, k-1 .
\end{aligned}\right.
$$

So we have

$$
X_{k}=Y_{k} H_{k} Z_{k}
$$

with $H_{k}=\operatorname{diag}\left(\left(\beta_{1}-\alpha_{1}\right) I_{r}, \cdots,\left(\beta_{k}-\alpha_{k}\right) I_{r}\right)$.

The agorithm is summarized as follows.

Agorithm II.1. LR-ADI method for (1) with $E=F G$.

Input: $A, D \in \square^{n \times n}, B, C \in \square^{m \times m}, F \in \square^{n \times r}$ and $G \in \square^{r \times m}$ with $\lambda D-A$ and $\lambda C-B$ are c-stable. The number of ADI steps $k$ and the ADI shifts $\left\{\alpha_{1}, \alpha_{2}, \cdots, \alpha_{k}\right\}$ and $\left\{\beta_{1}, \beta_{2}, \cdots, \beta_{k}\right\}$.

Output: $Y, H$, and $Z$ such that $X=Y H Z$ is an approximate solution of the projected generalized Sylvester equation (1) with $E=F G$.

1. Set 
2. For $i=1,2, \cdots$

$$
\begin{gathered}
Y_{(:, 1: r)}=\left(D-\beta_{1} A\right)^{-1} P_{l, 1} F, \\
Z_{(1: r,:)}=G P_{r, 2}\left(C+\alpha_{1} B\right)^{-1}, \\
H_{(1: r, 1: r)}=\left(\beta_{1}-\alpha_{1}\right) I_{r} .
\end{gathered}
$$

$$
\begin{gathered}
Y_{(:, i r+1:(i+1) r)}=\left(D-\beta_{i+1} A\right)^{-1}\left(D-\alpha_{i} A\right) Y_{(:,(i-1) r+1: i r)}, \\
Z_{(i r+1:(i+1) r,:)}=Z_{((i-1) r+1: i r,:)}\left(C+\beta_{i} B\right)\left(C+\alpha_{i+1} B\right)^{-1}, \\
H_{(i r+1:(i+1) r, i r+1:(i+1) r)}=\left(\beta_{i+1}-\alpha_{i+1}\right) I_{r} .
\end{gathered}
$$

End For

Since the computation of the Weierstrass canonical form is sensitive under small perturbations, we should make use of the generalized real Schur factorization to compute the spectral projectors, see, for example, [7,11]. For large-scale problems, the computation of the spectral projectors by the generalized real Schur factorization may be very expensive. However, in some applications the spectral projectors can be expressed in explicit form by using the special block structure of the matrices $A, D, B$ and $C$, see numerical examples in this paper or the reference [9].

If the number of shift parameters $k$ is smaller than the number of iterations required to obtain a prescribed tolerance, then we reuse these parameters in a cyclic manner. For the computation of shift parameters, we refer to $[8]$.

\section{Numerical experiments}

In this section, we present a numerical example to illustrate the performance of the LR-ADI method for the projected generalized Sylvester equation (1). In the following example, we compare the numerical behavior of LR-ADI with MGSIGN [10] with respect to the number of iterations (ITER), CPU time (CPU) and the relative residuals $\left\|R_{k}\right\|_{F} /\left\|R_{0}\right\|_{F}$ (RES).

All numerical experiments are performed on a PC with the usual double precision, where the floating point relative accuracy is $2.22 \times 10^{-16}$.

The stopping criterion for both methods is

$$
\frac{\left\|R_{k}\right\|_{F}}{\left\|R_{0}\right\|_{F}}=\frac{\left\|A X_{k} C+D X_{k} B+P_{l, 1} E P_{r, 2}\right\|_{F}}{\left\|P_{l, 1} E P_{r, 2}\right\|_{F}}<10^{-12} .
$$

We consider the 2D instationary Stokes equation that describes the flow of an incompressible fluid in a domain. The spatial discretization of this equation by the finite difference method on a uniform staggered grid leads to the descriptor systems

$$
\begin{aligned}
D \dot{x}(t) & =A x(t)+F u(t), \\
y(t) & =G x(t),
\end{aligned}
$$

and

$$
\begin{aligned}
C \dot{x}(t) & =B x(t)+F u(t), \\
y(t) & =G x(t) .
\end{aligned}
$$


The matrix coefficients in (15) is given by

$$
D=\left[\begin{array}{ll}
I & 0 \\
0 & 0
\end{array}\right] \in R^{n \times n}, \quad A=\left[\begin{array}{cc}
A_{11} & A_{12} \\
A_{21} & 0
\end{array}\right] \in R^{n \times n} .
$$

These matrices are sparse and have a special block structure. Using this structure, the projectors $P_{l, 1}$ and $P_{r, 1}$ onto the left and right deflating subspaces of the pencil $\lambda D-A$ can be computed as

$$
\begin{aligned}
& P_{l, 1}=\left[\begin{array}{ccc}
\Pi & -\Pi A_{11} A_{12}\left(A_{21} A_{12}\right)^{-1} \\
0 & 0
\end{array}\right], \\
& P_{r, 1}=\left[\begin{array}{cc}
\Pi & 0 \\
-\left(A_{21} A_{12}\right)^{-1} A_{21} A_{11} \Pi & 0
\end{array}\right],
\end{aligned}
$$

where $\Pi=I-A_{12}\left(A_{21} A_{12}\right)^{-1} A_{21}$ is the orthogonal projector onto the kernel of $A_{21}$ along the image of $A_{12}$, see $[8,12]$. The matrices $A_{12}$ and $A_{21}$ have full rank and the pencil $\lambda D-A$ is of index 2 . Analogously, we can get the projectors $P_{l, 2}$ and $P_{r, 2}$ of the pencil $\lambda B-C$. In our experiment the state space dimensions of the problems are $n=2295$ and $m=1975$, respectively. The matrix $E$ in (1) is $E=F G$ with $F \in \square^{2295 \times 1}$ and $G \in \square^{1 \times 1975}$.

The results in Table 2 clearly indicate that the LR-ADI method is more efficient than MGSIGN for this example according to the CPU time.

TABLE I

\begin{tabular}{|c|c|c|c|}
\hline & ITER & CPU & RES \\
\hline LR-ADI & 30 & 10.68 & $8.9 \times 10^{-13}$ \\
\hline MGSIGN & 15 & 296.57 & $7.4 \times 10^{-13}$ \\
\hline
\end{tabular}

\section{Conclusion}

In this paper, we have proposed the generalized low-rank alternating direction implicit method to solve the projected generalized Sylvester equations. Numerical experiments presented in this paper show the effectiveness of the proposed method.

\section{References}

[1] F. Gantmacher, Theory of Matrices, Chelsea, New York, 1959.

[2] B. Anderson and J. Moore, Optimal Control-Linear Quadratic Methods, Prentice-Hall, Englewood Cliffs, NJ, 1990.

[3] U. Helmke and J. Moore, Optimization and Dynamical Systems, Springer, London, 1994.

[4] V. Mehrmann, The Autonomous Linear Quadratic Control Problem, Theory and Numerical Solution, Lecture Notes in Control and Information Sciences, Vol. 163, Springer, Heidelberg, 1991.

[5] T. Stykel, "Gramian-based model reduction for descriptor systems", Math. Control Signals Systems, vol. 16, no. 4, pp. 297-319, May 2004. 
[6] T. Stykel, "Stability and inertia theorems for generalized Lyapunov equations", Linear Algebra Appl., vol. 355, no. 1, pp. 297-314, November 2002.

[7] T. Stykel, "Numerical solution and perturbation theory for generalized Lyapunov equations", Linear Algebra Appl., vol. 349, no. 1, pp. 155-185, July 2002.

[8] T. Stykel, "Low-rank iterative methods for projected generalized Lyapunov equations", Elect. Trans. Numer. Anal., vol. 30, no. 2, pp. 187-202, August, 2008.

[9] T. Stykel, "A modified matrix sign function method for projected Lyapunov equations", Systems Control Lett., vol. 56, no. 11, pp. 695-701, November 2007.

[10] Y. Lin, L. Bao and Y. Wei, "Matrix sign function methods for solving projected generalized continuoustime Sylvester equations", IEEE Trans. Auto. Contr., vol. 58, no. 11, pp. 2629-2634, November 2010.

[11] G. H. Golub and C. F. Van Loan, Matrix Computations, 3rd edition, Johns Hoplins University Press, Baltimore, 1996.

[12] J. Sabino, Solution of Large-Scale Lyapunov Equations via the Block Modified Smith Method, PhD thesis, Rice University, Houston, Texas, 2006. 